\title{
Discovering Gaps in Saudi Education for Digital Health Transformation
}

\author{
Adeeb Noor (iD) \\ Department of Information Technology \\ Faculty of Computing and Information Technology (FCIT) \\ King Abdulaziz University (KAU), Jeddah (80221), Kingdom of Saudi Arabia (KSA)
}

\begin{abstract}
The growing complexity of healthcare systems worldwide and the medical profession's increasing dependency on information technology for accurate practice and treatment call for specific standardized education in health informatics programming, and accreditation of health informatics programs based on core competencies is progressing at an international level. This study investigates the state of affairs in health informatics programs within the Kingdom of Saudi Arabia (KSA) to determine (1) how well international standards are being met and (2) what further development is needed in light of KSA's recent eHealth initiatives. This descriptive study collected data from publicly available resources to investigate Health Informatics programs at 109 Saudi institutions. Information about coursework offered at each institution was compared with American Medical Informatics Association (AMIA) curriculum guidelines. Of 109 institutions surveyed, only a handful offered programs specifically in health informatics. Of these, most programs did not match the coursework recommended by AMIA, and the majority of programs mimicked existing curricula from other countries rather than addressing unique Saudi conditions. Education in health informatics in KSA appears to be scattered, non-standardized, and somewhat outdated. Based on these findings, there is a clear opportunity for greater focus on core competencies within health informatics programs. The Saudi digital transformation (eHealth) initiative, as part of Saudi Vision 2030, clearly calls for implementation of internationally accepted health informatics competencies in education programs and healthcare practice, which can only occur through greater collaboration between medical and technology educators and strategic partnerships with companies, medical centers, and governmental institutions.
\end{abstract}

Keywords-Health informatics; education; information technology; American Medical Informatics Association (AMIA); Saudi Arabia; vision 2030

\section{INTRODUCTION}

The professions of healthcare cover a vast and growing number of fields, many of which are ever evolving and increasingly interdisciplinary. The broad span of healthcare requires a complex conglomeration of skills and knowledge that both generates and utilizes a staggering network of biomedical data for the study, research, analysis, and administration of our physical quality of life. The management and leveraging of these resources has led to the developing interdisciplinary field of health informatics [1,2]. One of the challenges to this development lies in addressing the ambiguity and sheer breadth of health informatics, which is referenced in literature and in practice under many names and categories, including medical informatics, medical information technology, and others. Several organizations have created a spectrum of subcategories in an attempt to classify the many faces of health informatics, with considerable emphasis on establishing and maintaining standards of education.

In comparing curricula of biomedical and health informatics programs in the United States, Kampov-Polevoi and Hemminger [3] determined that programs varied widely in terms of the professional competencies required for health informatics and in the key content of supporting curricula. This gap provided the impetus for their research, which attempted to provide a holistic view of current educational options. Kampov-Polevoi and Hemminger also acknowledged that, at the time of their study, the debate surrounding the definition of the fields of biomedical and healthcare informatics had already spanned decades [4-11]. By 2012 the effects of this identity confusion finally became a priority for those seeking to clarify the competencies needed for accreditation in these fields.

In 2012, a white paper from the American Medical Informatics Association (AMIA) [1] outlined an initial set of competencies to serve as the basis for collaboration with the Commission on Accreditation for Health Informatics and Information Management (CAHIIM) in 2015. Out of this collaboration, the Health Informatics Accreditation Council (HIAC) was born, an organization whose mandate was to set "Accreditation Standards for Masters' Degree Programs in Health Informatics." This process resulted in the establishment of a set of foundational domains defining health informatics. These efforts of AMIA and other members of the International Medical Informatics Association (IMIA) have attempted to standardize programs of study in health informatics on an international level. However, colleges and universities have historically developed their curricula based on internal decision-making processes, not necessarily based on the recommendations and practices of outside organizations, whether academic, industrial, or governmental.

As our world continues to evolve technologically, politically, and in size of population, standardizations in the management of healthcare systems are becoming increasingly critical. The days of small clinics and independent hospitals with extremely proprietary filing systems are approaching extinction. With the growing complexity and variety of methods, treatments, services, and equipment, the breadth and 
complexity of the corresponding informatics for managing this growth naturally follows closely behind. As is the case with most changes at the national level in any country, the adaptation of the healthcare industry varies by culture and established institutions and practices.

From these observations in the international environment, we shift our focus specifically to the current state of affairs for obtaining education in health informatics in the Kingdom of Saudi Arabia (KSA). Understanding the challenges faced by the Saudi government in implementing nationwide standards of education in health informatics provides a perspective that could have broader implications for international education in this field. A study of these challenges could provide insight to students seeking technical education for healthcare fields, institutions working toward accreditation, researchers tracking the progress of development in the field of health informatics, and administrators seeking guidance for addressing the current state of affairs in their own contexts. Additionally, development of educational programs in health informatics across Saudi Arabia fits squarely within the purview of the eHealth strategy of the Saudi Ministry of Health (MOH) because of the government's Saudi Vision 2030 initiative to achieve digital transformation in healthcare. A recent study shows a strong need for E-health systems to be integrated on a national level [12], thus highlighting the demand for qualified new graduates who can lead this transformation.

The aim of this paper is to study the importance of education standards in achieving health digital transformation specifically in the KSA. First, the paper begins with a literature review of related work (Section II), followed by a description of the methods used (Section III) and results found (Section IV). The paper concludes with discussion of findings and challenges in the evaluation of KSA's health informatics programs (Section V) and suggestions for curriculum improvements and future research (Section VI).

\section{RELATED WORK}

Several studies have already begun exploring the context of Saudi health informatics education. Altwaijiri and Aldosari [13] published a paper proposing the creation of a master's program adaptation based on the programs of three universities in the USA, Canada, and Australia. While this effort was a notable and organized attempt at developing health informatics programs for a single university, the approach taken was one of wholesale adoption and adaptation of targeted foreign programs rather than identifying and weighing specific core competencies for robust development and future-proofing. Four other works [14-17] have demonstrated the importance of health informatics in learning and decision-making but without focusing on the development of specific health informatics programs. Asiri [18] published a brief overview of some health informatics programs offered by Saudi educational institutions and attempted to address the challenges faced in the KSA and the international community as health informatics education is improved. These efforts to address and advise based on current research are laudable, though there is still room for other perspectives and no definitive solutions have yet surfaced. Fortunately, awareness is growing. Fallatah [19] published a work focusing on the need for collaboration and teamwork as a part of interprofessional education (IPE) in order to prevent medical errors and promote the advancement of healthcare in the KSA. Another recent work suggests that the Saudi Health Informatics Competency Framework (SHICF) could be used to establish health informatics standards for the kingdom [20].

This interdisciplinary and collaborative aspect of healthcare education, already well-recognized in the west, is beginning to make its way into the curricular programs of the KSA. By focusing on these programs, the following research provides a rapid, precise, and professional vision for those with a vested interest in health digital transformation development, not only in Saudi Arabia but also in other Arab and developing nations.

\section{METHODS}

As with the ambiguities that have persisted in classifying and standardizing the skills and education of the fields of health informatics on an international level, ambiguities also exist when reviewing related educational programs and courses within the KSA. In an attempt to clarify some of these ambiguities, the Faculty of Computing and Information Technology at King Abdulaziz University conducted a descriptive survey in August 2018 to learn about the structure of educational programs and availability of key courses at schools listed by the Saudi Ministry of Education (MOE). This work is the exposition and analysis of the findings of that survey.

Public information sources were used whenever possible in order to replicate the experience of a layperson seeking knowledge through government resources, official websites, information published (officially or tangentially) on the public internet, and direct email and voice communications with those officially involved in the administration of programs and courses. Searches were carefully limited to reliable channels that would be accessible and comprehensible to persons with a moderate understanding of the nature and fields of health informatics, such as potential students or non-specialized professionals seeking information about educational offerings or trying to evaluate the merits of enrollment. Thus, initial internet data was collected through use of a simple Boolean logic search ([URL of the University] OR [Name of the University in Arabic] OR [Name of the University in English]) AND (health Informatics OR medical Informatics OR bioinformatics)) was used. When the Boolean search did not return information about health informatics programs at a given institution, the researchers contacted institutional administrators directly in order to ascertain whether the university, in fact, offered no health informatics coursework or whether these courses were simply not included as a part of the institution's online presence.

Using these methods, a sample was collected consisting of information about programs and courses at 109 colleges and universities located throughout the KSA, including public, private, and military schools. For those schools listing specific programs in health informatics, the availability of course offerings matching the AMIA recommendations were also examined. 


\section{RESULTS}

For the current research, determination of the origins and motivations for existing programs and courses specific to health informatics proved inconclusive due to the lack of information made publicly available by the surveyed academic institutions. Only 10 of the 109 academic institutions surveyed openly offered programs specific to health informatics, with most of these being bachelor's degree programs. Programs related to medical coding appeared to be disproportionately prevalent. This is most likely because medical coding is considered a certification path rather than simply one course offered within a curriculum. For the programs specifically pertaining to "Health Informatics", all 109 academic institutions were considered by region and by type in order to provide a geographic and political breakdown.

Fig. 1 shows a geographic breakdown indicating that the number of surveyed academic institutions located in the central and western regions of the KSA dominated more than $50 \%$ of the sample.

This is unsurprising as those regions include the cities of Riyadh, Jeddah, Madinah, and Makkah, which have longstanding academic institutions. However, Fig. 2 shows that surveyed academic institutions with specific health informatics programs were concentrated in the central and eastern regions of the KSA with $80 \%$ of the sample.

Fig. 3 shows a political breakdown by type of academic institution and indicates that the surveyed academic institutions were overwhelmingly public by a nearly 3 to 1 margin over the private institutions and an 11 to 1 margin over the military institutions.

In even greater relief, Fig. 4 shows that 4 out of 5 of the surveyed academic institutions with specific health informatics programs were public with military institutions having no showing at all. As previously mentioned, out of the 109 surveyed institutions, only 10 had programs specifically labeled as "health informatics". Information was gathered regarding the curricula of these 10 surveyed programs regarding the similarity of their courses to 22 courses recommended by AMIA for health informatics programs.

This review only took into account whether a comparable course was offered in each program. It was found that on average each program offered half of the 22 courses, although which of the 22 courses varied considerably by program. Only 6 of the 22 recommended courses were offered in 7 or more programs. The minimum number of the recommended courses offered by any program was 3 , while the maximum number offered by any one program was 20. These minimal and maximal cases were extreme outliers with all remaining numbers of courses being heavily concentrated between 7 and 14. Table I demonstrates the list of courses along with an indication of how many of the 10 universities offered each course.

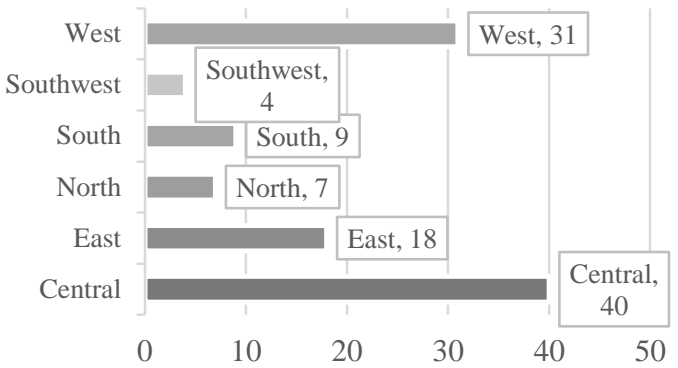

Fig. 1. Number and Proportion of Schools Aurveyed by Region.

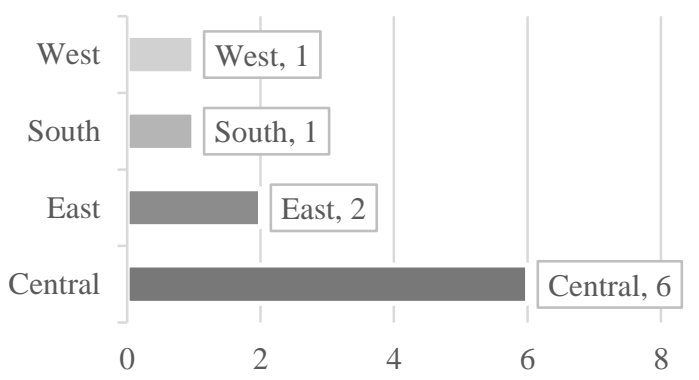

Fig. 2. Number and Proportion of Schools Aurveyed with a Health Informatics Program by Region.

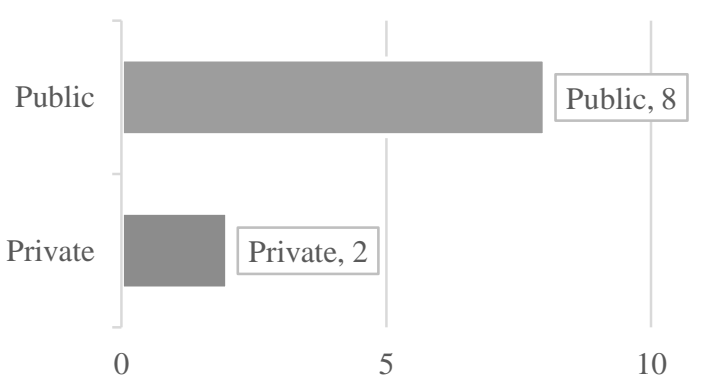

Fig. 3. Number and Proportion of Schools Surveyed by Type.

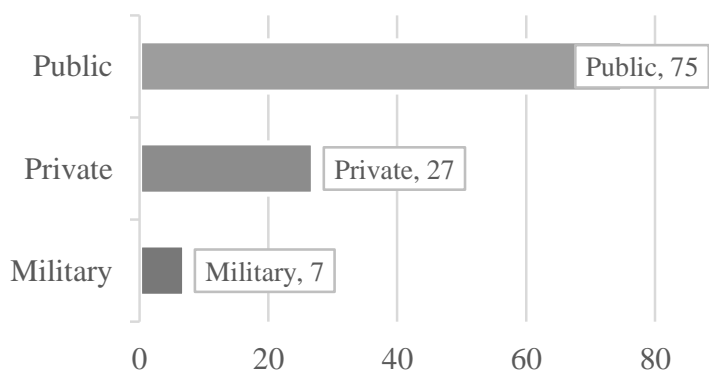

Fig. 4. Number and Proportion of Schools Surveyed with a Health Informatics Program by Type. 
TABLE. I. COURSES Recommended by the AMERICAN Medical INFORMATICS ASSOCIATION ${ }^{3}$

\begin{tabular}{|l|l|}
\hline Name of course & Occurrences out of 10 universities \\
\hline Introduction to Health Informatics & 5 \\
\hline Public Health Informatics & 6 \\
\hline Electronic Health Records & 5 \\
\hline Coding System & 7 \\
\hline Research Methodology & 8 \\
\hline Epidemiology & 8 \\
\hline Biostatistics & 7 \\
\hline Data Mining & 3 \\
\hline Data Warehousing & 3 \\
\hline Decision Support System & 5 \\
\hline System Analysis and Design & 5 \\
\hline Computer Programing & 4 \\
\hline Database Management Systems & 7 \\
\hline Information Security & 6 \\
\hline Human Computer Interaction & 1 \\
\hline Health Promotion and Education & 4 \\
\hline Global Health & 3 \\
\hline Legal and Ethics in Health Informatics & 9 \\
\hline Health Economics & 4 \\
\hline Interdisplinary Perspectives & 1 \\
\hline Organization Behaviors & 5 \\
\hline Leadership in Healthcare & 2 \\
\hline
\end{tabular}

\section{DISCUSSION}

Although the findings seem to show clear gaps in health informatics education in the KSA, several limitations of the research must be noted. One limitation was the use of common Boolean search patterns for gathering internet-based information. The breadth of competencies that fit into the interdisciplinary field of health informatics is vast and often difficult to specifically label as pertinent to health informatics. The ever-evolving nature of health informatics, particularly regarding education and training, is still being defined.

In addition, because health informatics is referred to by many different names and can be housed in many different academic departments, the Boolean search did not always yield complete results, even when related courses existed. Health Informatics programs were offered within various departments, such as medical or computer science departments, with little indication as to where to search. This is likely due to the interdisciplinary nature of the field and the lack of attribution of data-related and analysis courses specifically pertinent to health informatics. Many of the websites of the academic institutions did not provide specific descriptions of the courses offered within their departments. Offline efforts to inquire further into the programs did not usually yield any additional information. These outcomes are not necessarily specific to health informatics but are potentially exacerbated by lack of awareness and focus on health informatics at the departmental level. In short, programs and courses exist, but finding them and specifically identifying them proved difficult, thus showing that popularity and awareness of health informatics fields is not yet pervasive. To help address this, there is an ongoing annual conference for health informatics (http://www.saudi-ishic.com) directed at increasing awareness in the KSA.
From the onset of the survey, attempts to inquire into programs and courses related to health informatics for the 109 colleges and universities throughout the KSA proved difficult. A vast array of fields and subcategories fall within the scope of health informatics with most institutions either not providing any specifics or focus in fields related to health informatics or only referencing specifics when the nature of the course was strongly oriented towards a specific field. For example, Research Chair for Health Informatics (http://rchip.org) was found to be a specialized training program specific to health informatics. However, the lack of specification for health informatics programs and courses appeared to be greater than the inconsistencies noticed in documentation of other programs and courses in more traditional fields.

At some universities, health informatics coursework was offered through medical or healthcare departments, whereas at other institutions it was housed under the umbrella of information technology or computer science. To further complicate matters, academic programs at Saudi universities are not always well documented. Some institutions do not offer specific course descriptions on their websites, necessitating direct communication with faculty and administrators in order to determine the nature of coursework within their departments. These communications sometimes provided inaccurate or inconsistent information or referrals. For instance, officials in some cases communicated that existing programs did not exist or made referrals to incorrect contacts or departments at the institution. These situations presented significant challenges for gathering consistent information about each school's health informatics offerings.

For these and other reasons, names of specific institutions have not been mentioned in this paper. This is to prevent undue criticism where such criticism may not be warranted. In many cases, health informatics may not have matched the primary focus of an institution, while in other situations the very changing nature of the health informatics field presented challenges in documenting exactly what training was being provided.

Despite efforts to standardize accreditation at the international level through the IMIA, of which the KSA is a member via the Saudi Association for Health Informatics (SAHI), the effects of such standardizations require prioritization and time. As health informatics is a stillevolving collection of fields, skills, and knowledge, it is necessary for the institutions of education to be constantly vigilant and to remain apprised of current best practices. Unfortunately, at the time of writing of this manuscript, the SAHI website (http://www.sahi.org.sa/) was advertising conferences and seminars for 2015 and 2017, nearly four and two years out of date. Clearly, if the focus on events provided by the national representative of the KSA in IMIA is this outdated, this does not bode well for the programs and courses of the educational institutions of the KSA, which take far more time to develop and update. This observation is also reinforced by the lack of published literature regarding health informatics programs in the KSA. Hopefully, the information gathered and surveyed for this work regarding existing curricula and programs will contribute to filling this gap and 
provide greater focus on the state of Saudi health informatics education.

\section{CONCLUSION}

While standardization efforts have been made at the international level for identifying and classifying core competencies in health informatics at the graduate level, this is an ongoing process due to the relative newness of the discipline as well as the ever-changing skills and knowledge associated with managing and maintaining the well-being of humanity. With each nation adopting and interpreting courses and programs pertaining to health informatics in its own academic institutions, this study has found that there is great room for advancement in standardizing accredited programs and courses in the field of health informatics in the Kingdom of Saudi Arabia. The general lack of information and specifics about health informatics programs found from surveying 109 universities and colleges in the KSA is due partly to incomplete or missing descriptions of courses and programs on websites and other materials made available to the public. It was not usually apparent which department or program to search for when looking for this information, and attempts to contact departments for additional information were often misleading or inconclusive.

If health informatics education in KSA is to improve, research must be prioritized at both academic and national levels, especially in understanding core competencies within the fields of health informatics. Out of the 109 institutions surveyed in this research, only 10 offered specific programs in health informatics, and those programs offering, on average, only half of the 22 courses recommended by the AMIA. Is this because international standards do not match the needs of KSA healthcare programs or facilities? Is more financial investment in health technology needed, or is the critical issue one of human resources and better training in the use and teaching of health informatics? These questions can be answered through further research and focused development of KSA's health informatics education programs.

To support this research, the Ministries of Health and Education may need to not only augment their own collaboration but also forge new strategic partnerships with companies, medical centers, and governmental institutions, all with the objective of promoting specific educational programs in health informatics that will lead to practical healthcare applications. Such partnerships would provide opportunity to develop benchmark programs for health digital transformation within the Arab World and developing nations, realizing the MOH's eHealth initiative and helping to achieve Saudi Vision 2030.

Declaration of Interest--The authors report no declarations of interest.

\section{ACKNOWLEDGMENTS}

We acknowledge support from King Abdulaziz University, Jeddah, Saudi Arabia.

\section{REFERENCES}

[1] Kulikowski, C., Shortliffe, E., Currie, L., Elkin, P., Hunter, L., Johnson, T., Kalet, I., Lenert, L., Musen, M., Ozbolt, J., Smith, J., TarczyHornoch, P. and Williamson, J. AMIA Board white paper: "Definition of biomedical informatics and specification of core competencies for graduate education in the discipline." J Am Med Inform Assn, vol. 19, no. 6 , pp.931-938, 2012.

[2] Fridsma, D. "The scope of health informatics and the Advanced Health Informatics Certification.” J Am Med Inform Assn, vol. 23, no. 4, pp.855-856, 2016.

[3] Kampov-Polevoi, J. and Hemminger, B. "A curricula-based comparison of biomedical and health informatics programs in the USA." J Am Med Inform Assn, vol. 18, no. 2, pp.195-202, 2011.

[4] Hasman, A., Haux, R. and Albert, A. "A systematic view on medical informatics."Comput Meth Prog Bio, vol. 51, no. 3, pp.131-139, 1996.

[5] Huerta, M., Downing, G., Haseltine, F., Seto, B., and Liu Y. "NIH working definition of bioinformatics and computational biology." US National Institute of Health, Jul 17, 2000.

[6] Miller, P. "Opportunities at the intersection of bioinformatics and health informatics: A case study."J Am Med Inform Assn, vol. 7, no. 5, pp.431-438, 2000.

[7] Mantas, J. and Hasman, A. Textbook in health informatics. Amsterdam: IOS Press. 2003.

[8] Staggers, N. "The evolution of definitions for nursing informatics: A critical analysis and revised definition." J Am Med Inform Assn, vol. 9, no. 3, pp.255-261, 2002.

[9] Shortliffe, E.H. and Blois, M.S. "The computer meets medicine and biology: emergence of a discipline." In Biomedical Informatics, pp. 345. Springer, New York, 2006.

[10] Gardner, R., Overhage, J., Steen, E., Munger, B., Holmes, J., Williamson, J. and Detmer, D. "Core content for the subspecialty of clinical informatics." J Am Med Inform Assn, vol. 16, no. 2, pp.153$157,2009$.

[11] Hersh, W. "A stimulus to define informatics and health information technology." BMC Med Inform Decis, vol. 9, no. 1, 2009.

[12] Noor, A. "The Utilization of E-health in the Kingdom of Saudi Arabia." International Research Journal of Engineering and Technology, vol. 6, no. 9, pp. 1229-1239, Sep, 2019.

[13] Altwaijiri, M. and Aldosari, B. "Health informatics Master program at King Saud bin Abdulaziz University for Health Sciences, Riyadh, Saudi Arabia."Yearbook of Medical Informatics, vol. 17, no. 1, pp.145-149, 2008.

[14] Albarrak, A. "Designing e-learning systems in medical education: A case study." International Journal of Excellence in Healthcare Management, vol. 183, no. 3119, pp. 1-9, Jun, 2010.

[15] Albarrak, A.I. "E-learning in medical education and blended learning approach." Learning, vol. 13, pp. 14-20, 2011.

[16] Househ, M.S., Saddik. B., and Al-Dosari. B. "Development of a graduate level course in e-health and emerging technology in Saudi Arabia." St Heal T, pp. 69-73, Feb 9, 2011.

[17] Bahkali, S., Almaiman, A., Almadani, W., Househ, M.S., and El Metwally, A. "The state public health informatics in Saudi Arabia." St Heal T, pp. 257-260, Jul 24, 2014.

[18] Asiri, H. A. "Challenges of the health informatics education in the Kingdom of Saudi Arabia: What stands in our way?" Journal of Health Informatics in Developing Countries, vol. 8, no. 1, Apr 8, 2011.

[19] Fallatah, H. "Introducing inter-professional education in curricula of Saudi health science schools: An educational projection of Saudi Vision 2030." Journal of Taibah University Medical Sciences, vol. 11, no. 6, pp.520-525, 2016.

[20] Almalki, M., Househ, M., and Alhefzi, M. "Developing a Saudi Health Informatics Competency Framework: A Comparative Assessment." St Heal T, vol. 264, pp. 1101-1105, 2019. 